\title{
Connaissance des produits agricoles
}

Marie-Jeanne HUSSET

60 Millions de consommateurs,

80 Rue Lecourbe,

75015 Paris

<mj.husset@inc60.fr>

Comme mes prédécesseurs, plus le colloque approchait plus je me suis dit : "Pourquoi ai-je accepté d'intervenir dans ce colloque? » En plus, que veut dire connaissance des produits agricoles? Et finalement pourquoi moi qui ne suis ni chercheur, ni sociologue, ni même (excusezmoi) représentante des associations de consommateurs? Je suis représentante de 60 millions de consommateurs. Cela veut dire modeste journaliste, directrice de la rédaction du magazine de l'Institut national de la consommation, magazine de service public, je le précise. Donc je ne représente pas les 18 associations de consommateurs agréées et $d$ 'ailleurs je serais bien en peine de les représenter car je ne sais pas si toutes ensemble elles ont une opinion commune sur ce qui nous rassemble aujourd'hui. Que savent les consommateurs des produits agricoles? J'ai commencé par me dire: "C'est ce qu'on leur a appris à l'école, ce qu'ils lisent dans les journaux, ce qu'ils voient sur les étiquettes. » Peut-être d'ailleurs qu'ils ne voient pas grand-chose. On va $y$ revenir.

Mais avant, peut-être parce que justement je suis journaliste, j'aime bien asticoter. II me semble que mon rôle est plus de lancer des pistes de réflexion et de débat. Je voudrais d'abord vous faire part des résultats de deux enquêtes que nous avons publiées dans 60 millions de consommateurs en 2004. Cela semble dater un peu, mais je ne pense pas que les résultats seraient radicalement différents aujourd'hui.

La première enquête, nous l'avons publiée au moment d'un salon de l'agriculture en mars 2004, et nous l'avions faite en collaboration avec les chambres d'agriculture. Nous voulions savoir quelles étaient les relations entre les consommateurs et les agriculteurs. On est peut-être un peu dans le sujet qui nous préoccupe aujourd'hui. Nous avions demandé aux consommateurs: "Quelle est pour vous la première mission de l'agriculture?» Pour les consommateurs, la première mission de l'agriculture, et cela devrait être encore plus vrai aujourd'hui, c'est de nourrir la population. Cette réponse me semble importante, encore plus aujourd'hui où l'on voit se développer les

\author{
Abstract: For consumers, it's clear now that quality is a package that includes both the quality of \\ health, nutritional quality and taste. But what it's clear too too, that the consumers have a very few \\ knowledge of food. Informations through labelling for fresh products like processed products still need \\ to be completed. \\ Key words: consumer, quality, health, knowledge of food
}

agrocarburants et où justement il y a une concurrence entre les cultures à destination des carburants et les cultures vivrières. À un moment où on parle aussi beaucoup d'augmentation des prix des matières premières agricoles avec l'incidence que cela peut avoir sur le prix final, le prix au consommateur, je pense qu'avec les crises peut-être à venir en particulier sur les prix, il y a matière à réflexion, pas seulement au niveau français mais aussi au niveau européen et même international. Est-ce qu'au niveau international en tout cas nous ne sommes pas en train de nous tromper en poussant les agriculteurs et l'agriculture à délaisser un peu sa mission première, celle de nourrir l'humanité?

Autre question posée aux lecteurs pour essayer de déterminer les rapports entre les consommateurs et les agriculteurs: "Par rapport aux générations précédentes, pensez-vous que l'on mange plutôt moins bien ou plutôt mieux qu'avant ? » Et à $63 \%$ les répondants disaient qu'ils avaient l'impression qu'on mangeait plutôt moins bien qu'avant et quand on leur demandait ensuite : "A qui attribuez-vous cela, aux industriels, aux distributeurs, aux agriculteurs?", c'était d'abord aux industriels et ensuite aux distributeurs qu'ils attribuaient ce mauvais score et ils épargnaient les agriculteurs. Enfin, lorsqu'on leur demandait « concernant les conditions d'exercice de l'agriculture que jugez-vous le plus important? » ils mettaient en première position pour $89 \%$ d'entre eux, la protection de l'environnement. Vous voyez pourquoi je donne ces chiffres.

Autre enquête que nous avons menée.

Un peu à la fin de l'année 2004 nous avions essayé de cerner ce que, pour les consommateurs, bien manger veut dire. Nous avions fait à la fin des années 90 en plein milieu des crises alimentaires, avec le CREDOC, ce type d'enquête, pour essayer de cerner ce qu'était la qualité alimentaire. Alors que nous étions en plein dans les crises alimentaires, on voyait le bon sens des consommateurs français qui mettaient en premier critère de la qualité le goût et nous pensions : "Quand même les consommateurs ne se laissent pas impressionner par tout ce qui se passe, on est au pays de la gastronomie et finalement ils sont pleins de bon sens ces consommateurs. » Et puis quelques années après, au début des années 2000, on a commencé à voir des modifications. J'avais été présentée les résultats de cette enquête au CNA, Philippe Guérin s'en souvient peut-être, où j'avais suscité quelques réactions car j'avais commencé à parler d'américanisation de la société française puisqu'avant de leur demander: "Qu'est-ce que bien manger pour vous? " nous demandions "Comment mangez-vous ? ", et on commençait à apercevoir que les Français commençaient à manger de moins en moins à table, de plus en plus devant la télévision, de plus en plus en grignotant plusieurs fois dans la journée, etc. Bien manger pour les consommateurs - du moins selon notre enquête - c'était manger des fruits et des légumes. On peut penser au matraquage du PNNS, mais nous sommes en 2004, imaginez ce que ce doit être aujourd'hui, ce n'est pas $96 \%$, c'est $99,99 \%$ qui répondraient "c'est manger des fruits et des légumes." "À quel élément accordez-vous le plus d'importance?", ils répondaient «la qualité alimentaire » et lorsqu'on demandait " et la qualité qu'est-ce que c'est?", ils répondaient "c'est la fraîcheur ». Autrement dit, plus les consommateurs consomment des produits transformés, plus ils gardent le fantasme d'une alimentation qui serait une alimentation à base de produits frais et de qualité, équilibrée, et le fantasme de la fraîcheur.

Ces éléments étant donnés, que peut connaître le consommateur des produits alimentaires, alors que 70 à $80 \%$ de son alimentation sont d'origine transformée? Et que peut-il connaître par l'étiquetage lorsqu'il achète ? Je ne parle pas des autres pistes pour avoir une connaissance des produits alimentaires que sont la publicité, la communication, l'information plus ou moins bien donnée par la presse, ce qu'il apprend à l'école, etc. Pour les consommateurs, consommer c'est choisir, choisir c'est comparer. Mais que compare le consommateur? II compare le prix et il compare la qualité, c'est le fameux rapport qualité/prix si cher au consommateur sauf que c'est la notion de qua- 
lité qui importe et qui évolue pour les consommateurs au fur et à mesure que les périodes se succèdent. Ce qui est clair c'est que maintenant, pour les consommateurs, la qualité est un ensemble qui comprend à la fois la qualité sanitaire, la qualité nutritionnelle et la qualité gustative. On peut les mettre dans n'importe quel sens, peu importe. Je me souviens d'un débat que j'avais eu à un salon de l'agriculture avec un ministre de la Consommation ou un secrétaire d'Etat, au moment de l'affaire du poulet à la dioxine. II disait : "C'est de la faute des consommateurs, ils veulent le beurre et l'argent $d u$ beurre, ils voudraient avoir des produits de qualité et à bas prix. » Donc il séparait d'un côté la qualité, de l'autre la sécurité sanitaire. Plus personne n'oserait aujourd'hui défendre cette idée. Effectivement la sécurité sanitaire est un socle qui doit être le même pour tous les produits quel que soit le prix, mais qu'est-ce que la qualité ? Les consommateurs ne peuvent pas avoir le beurre et l'argent du beurre, et cela m'a donné l'idée de définir les consommateurs en disant que ce sont les seuls qui ont le droit de réclamer le beurre et l'argent du beurre. Cela veut dire des produits de qualité à des prix corrects. Après tout, pourquoi n'assignerait-on pas à la recherche, en tout cas à I'INRA, la mission de proposer aux consommateurs des produits de qualité à des prix relativement bas pour pouvoir donner un accès à la qualité à tous les consommateurs? La qualité c'est de toutes les façons ce triptyque mais aussi de plus en plus, c'est vrai pour I'alimentation mais aussi pour bien d'autres produits, ce que l'on pourrait appeler la qualité environnementale et même la qualité sociale c'est-à-dire les conditions de travail dans les exploitations agricoles, dans les usines des fabricants ou des sous-traitants. C'est tout ce que l'on appelle la consommation éthique. L'exigence est en train de monter et c'est une demande forte même si elle s'exprime encore d'une manière confuse parce que les indicateurs manquent. La demande est là mais l'offre $n^{\prime} y$ est pas vraiment, mais on sent que cela va faire partie de la qualité.

Donc pour ce qui est de la connaissance, comment les consommateurs peuvent-ils se déterminer lorsqu'ils sont devant les rayons ? Quelle connaissance peuvent-ils avoir des produits alimentaires? Lorsqu'on regarde les produits frais, notamment les fruits et les légumes, ils $n^{\prime}$ ont comme information pour se déterminer qu'un calibre, à la rigueur une origine et un prix. Je parlais tout à I'heure des pêches, comment peuvent-ils savoir ce qu'est une bonne pêche avant de la manger? Qu'est-ce qui fait que telle pêche qui vaut tant le kilogramme, par rapport à telle autre qui vaut deux fois moins le kilogramme, est meilleure? On n'en sait strictement rien. Pour les melons, cela va un peu mieux car on a l'impression que maintenant c'est difficile d'en avoir des mauvais. Mais comment le sait-on? Les melons sont à peu près tous sucrés aujourd'hui, c'est bien, mais comment se déterminent les melons de "Machin ", les melons d'ailleurs, les melons de "Bidule »? Tout cela reste extrêmement empirique. C'est vrai pour quelques fruits et pour les légumes. On sait que les tomates en hiver, mieux vaut ne pas en acheter, mais à part cela, on a très peu d'informations sur la qualité des fruits et des légumes. C'est très difficile. Nous $n^{\prime}$ avons pas d'indicateurs sur les produits transformés, on y reviendra. Prenons les viandes, il a fallu la crise de la vache folle pour que l'on puisse avoir une indication de l'origine, mais cela concerne le bœuf et on se demande pourquoi ils ne font pas cela sur le porc et sur I'agneau. Qu'attendent-ils ? Une crise du porc, une crise de l'agneau, une crise du poulet? Pourquoi n'aurions-nous pas ce même type d'indications sur toutes les viandes? Et le pois- son? Nous avions titré à une époque, «le poisson muet comme une carpe", l'étiquette muette comme une carpe. Effectivement nous avons attendu des années et des années, que I'on indique au moins au consommateur s'il est d'élevage ou de pêche sauvage. Une réglementation est entrée en vigueur en 2002 et nous sommes censés disposer de cette information ainsi que de la localisation géographique de la zone de pêche. Ce n'est pas forcément ce qui va nous donner la qualité du poisson ou la fraîcheur mais c'est au moins une indication $d^{\prime}$ origine. Je ne sais pas si vous avez fait cette constatation, d'ailleurs à 60 millions de consommateurs nous allons sans doute à nouveau nous occuper des poissonniers car cela agace un peu de voir que la réglementation est assez peu observée, très peu de poissonniers respectent la réglementation et donnent au moins la différence entre le poisson de pêche et d'élevage et la zone géographique. Pour tous les produits transformés, je passe sur l'étiquetage des ingrédients, des allergènes. Puisque l'on parle de la qualité, entre les signes officiels auxquels on peut relativement se fier (agriculture biologique, etc.) quels autres signes officiels ? Je sais que les pouvoirs publics mettent les $A O C$ dans les signes de qualité mais c'est plutôt un signe $d^{\prime}$ 'origine. Pour le reste il y a une profusion de signes qui ne sont pas forcément de qualité et qui accroissent la confusion des consommateurs qui ne comprennent plus rien entre ce que les industriels veulent mettre, même comme faux labels, avec plus ou moins des signes marketing qui se confondent. Les consommateurs nous le disent d'ailleurs, ils ne savent plus très bien comment $s^{\prime} y$ retrouver.

Donc, pour résumer, ce que connaissent les consommateurs des produits agricoles, en tout cas par l'information qui leur est donnée pour I'instant, je dirais que ce n'est pas grandchose. 